\title{
Circadian effect on steady-state pupil size is time of a day dependent
}

\author{
Feng Rao ${ }^{1}$, AHS Chan ${ }^{2}$ and Xi-fang $\mathrm{Zhu}^{3 *}$ \\ ${ }^{1}$ East China University of Science and Technology, Shanghai, PR China \\ ${ }^{2}$ Department of Systems Engineering and Engineering Management, City University of Hong Kong, Kowloon Tong, Hong Kong, PR China \\ ${ }^{3}$ School of Electrical and Optoelectronic Engineering, Changzhou Institute of Technology, Changzhou Jiangsu, PR China
}

\begin{abstract}
The present study investigated the time of day effect on steady-state pupil size induced by cirtopic effect. Two kinds of lights, a phosphor converted LED and a RGB LED, with the same photopic luminance and color temperature but different cirtopic luminance were chosen as the experimental light sources. The cirtopic effect induced by these two light sources was characterized by steady-state pupil diameter. Twenty healthy volunteers were tested at 09:00,15:00, 21:00 and 02:00 for-five working days. It was found that the cirtopic effect on pupil size was not statistically different in day time, but significantly different in night time, especially in midnight. Therefore, cirtopic effect on pupil size was time of day dependent.
\end{abstract}

\section{Introduction}

A number of studies have been conducted to develop the mechanism and equations between pupil size and luminance $[1,2]$. The discovery of a new class of photosensitive cells in the retina, the intrinsically photosensitive retinal ganglion cells (ipRGCs), which send retinal information induced by ambient light to the brain center to partly control the physiological characteristics of human, the pupil size, leads researchers to explore how the ipRGCs react under different luminance conditions. It is evidenced that the pupil size, an important factor of visual performance, is influenced by this cirtopic effect $[3,4]$. However, all past investigations of cirtopic effect on pupil size were conducted at a certain time of the day by researchers $[5,6]$. Whether the cirtopic effect on pupil size depends on time of a day has not been examined yet. In this study, the pupil diameters at different time of a day were measured to deduce the relationship between cirtopic effect on pupil size and time of a day.

Twenty university students, ranging in age from 18 to 23 years (mean $=21$ ) voluntarily participated in this study. The aims and procedures of the experiment were told to them and informed consent was given by them before testing. None of the participants had eye problems or general health complaints. All participants took part under the ethical guidelines of Changzhou Institute of Technology. The set-up of equipment was schematically illustrated in Figure 1. An integrating sphere of $50 \mathrm{~cm}$ diameter (Everfine Instrument Co. Ltd) was used to diffuse light rays equally. The voltage of a power supply could be regulated by two adjustable resistors for each of the two high power LEDs (a RGB LED and a phosphor converted LED) at a color temperature of $6500 \mathrm{~K}$.

The spectral distributions of the two LEDs are shown in Figure 2. The cirtopic action factors of luminous radiation [1] for the RGB LED and phosphor converted LED were1.1407 and 0.7034, respectively, which means the ratio of cirtopic luminance of RGB LED to phosphor converted LED with the same color temperature and photopic luminance is 1.1407:0.7034.
The participant looked at the opening of the sphere which had a diameter of $10 \mathrm{~cm}$. Viewing distance was $25 \mathrm{~cm}$, resulting in an angular extense of the illuminated circular field of $20 \mathrm{deg}$. Participants were asked to keep their chin on the chin rest to avoid head movements during the experiment. An infrared camera was used to capture videos of participant's right pupil at 30-frames-per-second. The ambient illuminance was lower than $0.5 \mathrm{~lx}$.

In each test, when the LEDs were switched on, the participant looked in to the opening with both eyes for $1 \mathrm{~min}$, and then the participant was asked to gaze at a target inside the sphere, and the pupil of the right eye was recorded with the infrared camera for $20 \mathrm{~s}$. The protocol of each test is shown in Figure 3. The luminance of the target inside the integrating sphere was kept $100 \mathrm{~cd} / \mathrm{m}^{2} \pm 5 \%$ (a common

\section{Target}

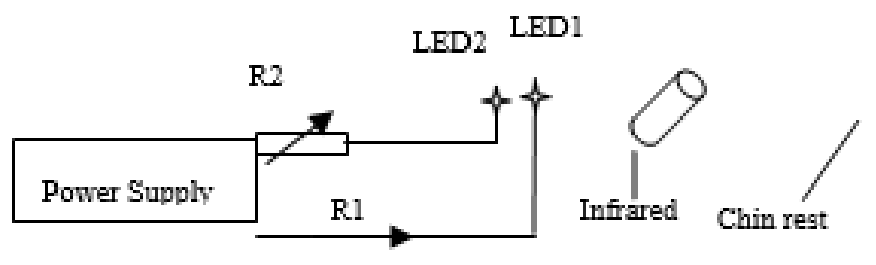

Figure 1. Schematic diagram of the test system

Correspondence to: Xi-fang Zhu, School of Electrical and Optoelectronic Engineering, Changzhou Institute of Technology, Changzhou Jiangsu 213002, PR China, E-mail: 1270437521@qq.com

Key words: pupil size, ipRGC, pupil light reflex, cirtopic

Received: November 02, 2017; Accepted: November 16, 2017; Published: November 20, 2017 
luminance of computer screen) through adjusting the resistor, and a color luminance meter (Everfine Instrument Co. Ltd, BM-9) was used to measure luminance every five minutes.

Before all the test sessions, a calibration was necessary to transform pixel values to linear values in millimeters by taking a photograph of ruler in front of eye, as shown in Figure 4.

The pupil size of a participant at the fifth, tenth and fifteenth second during the test time was measured. The mean pupil size values at the fifth, tenth and fifteenth second was used as representative values for examining the change of pupil size in this study. After completion of data collection, the pupil diameters of twenty participants against time of a day from Monday to Friday are plotted (see Figure 5). From the past researches, it was known that rods, cones and ipRGCs were all summed to influence the pupil size. In this paper, two lighting conditions with the same excitation of rods and cones but different ipRGCs excitation were employed. Therefore, the pupil functions only differed with the cirtopic effects in these two lighting conditions. The cirtiopic effect on pupil size can be characterized by the pupil size and whether it was time of day dependent can be obtained by comparing the pupil sizes in these two lighting conditions.

To investigate the strength of association of cirtopic effect on pupil size with time of a day, a student's t-test was performed on the pupil sizes under different lighting conditions, the $\mathrm{p}$ values with the $95 \%$ confidence interval are shown in Figure 5. A p value of less than $5 \%$ is always considered statistically significant. It was clear that the cirtopic effect on pupil size was statistically significant at 21:00 and 2:00, especially 2:00. No significant difference was shown in day time.

Pupil size of different lighting conditions against time of day. The blue dots and the stars represented the pupil diameters under RGB LED and phosphor converted LED lighting conditions, respectively at 9:00 condition. Symbols of other three colors are used to show the values at other time conditions $(15: 00,21: 00$, and 2:00). The empty squares and the circles indicated their mean values, the error bars indicate \pm 1 standard deviation (Figure 5).

Pupil size is very important for visual quality, the property of pupil can balance the ocular aberrations and retinal illuminance to obtain

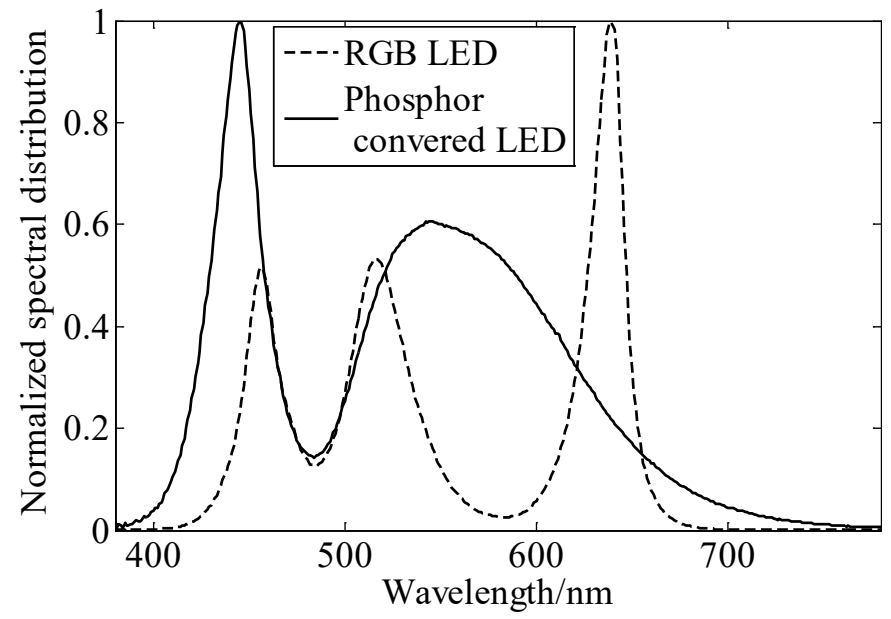

Figure 2. Spectral distributions of the two LEDs

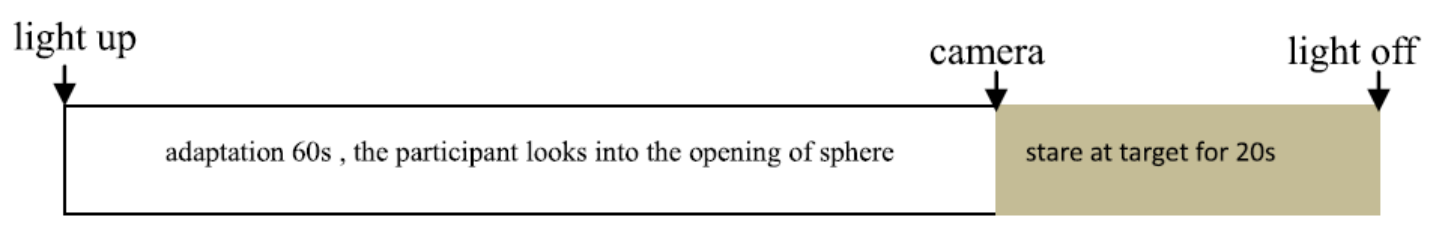

Figure 3. Protocol of a test

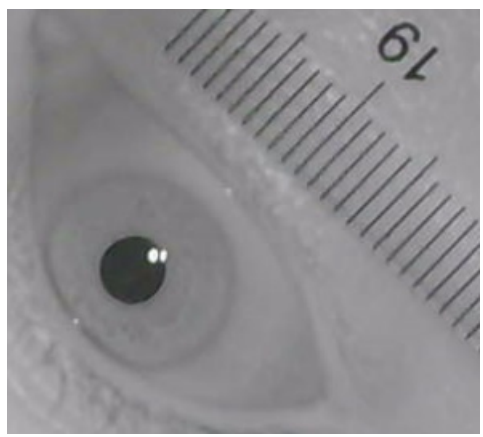

Figure 4. Calibration 


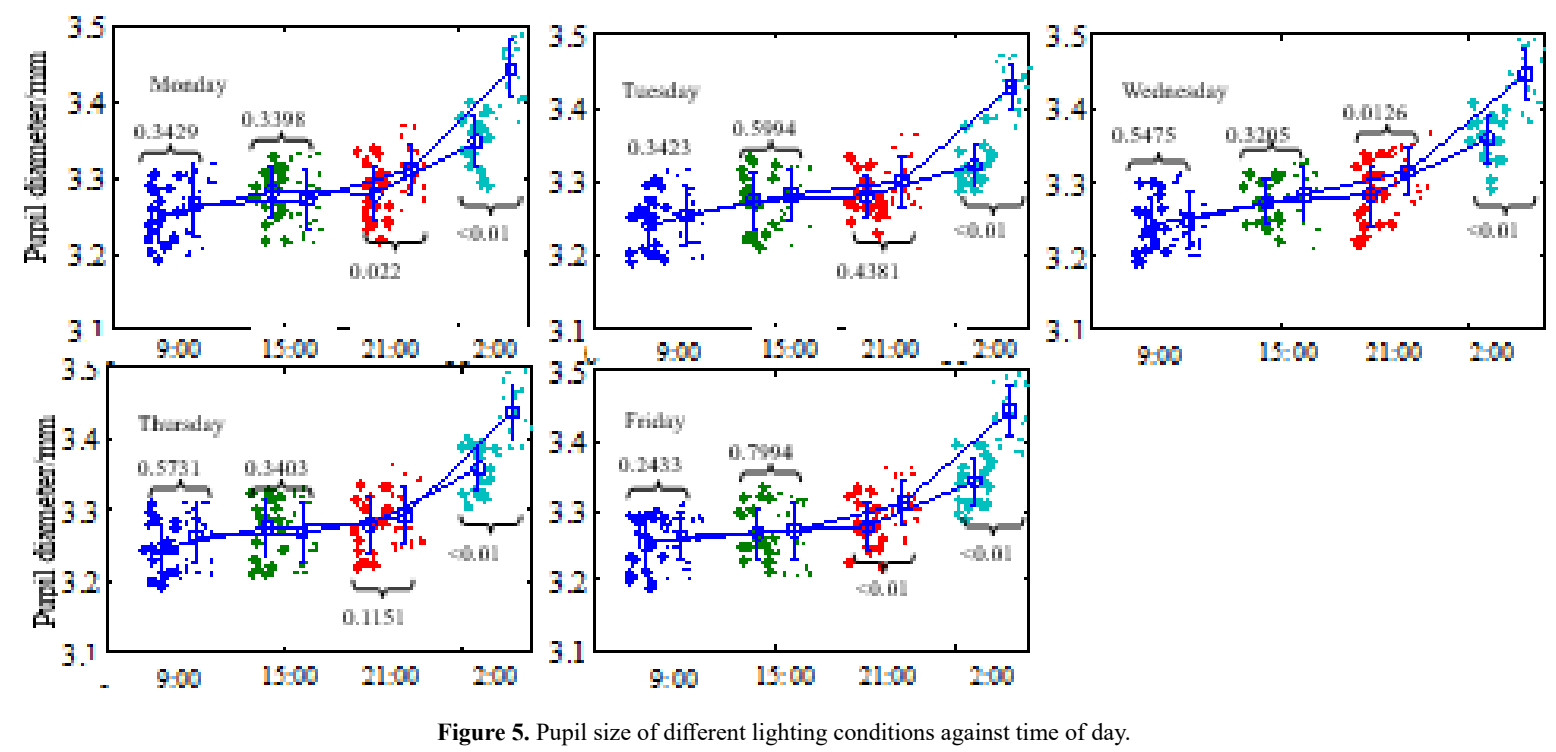

good vision quality [7]. From our research, it is clear the lighting conditions should be designed scientifically based on cirtopic effect and photopic vision for night worker while photopic theory may be enough for the day time situation.

In this paper, the pupil sizes were measured under the lighting conditions with the same photopic effect but different cirtopic effect, the cirtopic effect on pupil size against time of day was deduced through statistical analysis. It was clear that this effect was time of day dependent. These results are very important for the lighting design for night worker.

\section{Declaration of interest}

The authors report no conflicts of interest. The authors alone are responsible for the content and writing of the paper.

\section{Acknowledgements}

This research was supported by the National Key Research and Development Program of China (No:2017YFF0210000), Science and Technology Support Program me of Changzhou City(No:CE20055053), and the Overseas Training Program for
Outstanding Young Teachers and Principals of Universities of Jiangsu Province.

\section{References}

1. Schlangen LJM, Verhaegh J, Denissen AJM, Talen HJ , Herremans HML, et al. (2015) Workplace illumination effect on acuity, cognitive performance and well-being in older and young people. Proceedings of 28th CIE Session 87-95.

2. QingWanga, HaisongXu, RuiGong, JianqiCai. (2015) Investigation of visual fatigue under LED lighting based on readingtask. Optik 126: 1433-1438.

3. Rao F, Chan AHS, Zhu XF (2017) Effects of photopic and cirtopic illumination on steady state pupil sizes. Vision Res 137: 24-28. [Crossref]

4. Watson AB, Yellott JI (2012) A unified formula for light-adapted pupil size. J Vis 12 12. [Crossref]

5. Govén Tommy, Laike Thorbjörn, Raynham Peter, Sansal, Eren (2010) The influence of ambient lighting on pupils in classrooms considering visual, biological and emotional aspects as well as use of energy. Proceedings of the International Commission on Illumination Conference, Vienna, Austria, , 1-8

6. Xu W (2011) Research on non-visual photosensitive system based on pupil contraction $\mathrm{Ph}$.D. Dissertation in Optical Engineering.Fudan University. Jun. 2011

7. Wang W, Wang Y, Zhao KX (2010) The aberration and the modulation transfer function in LASEK and LASIK: Pupil size dependence. Optik, 121: 500-505

Copyright: (C2017 Rao F. This is an open-access article distributed under the terms of the Creative Commons Attribution License, which permits unrestricted use, distribution, and reproduction in any medium, provided the original author and source are credited. 